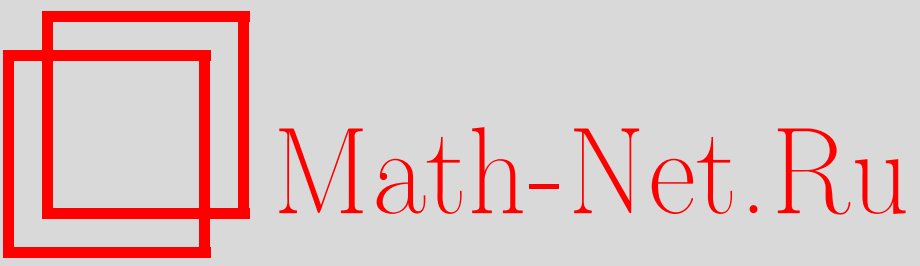

Г. Фальки, Ф. Магри, Г. Тондо, Редукция бигамильтоновых систем и разделение переменных: пример из иерархии Буссинеска, ТМФ, 2000, том 122, номер 2, 212 230

DOI: https://doi.org/10.4213/tmf564

Использование Общероссийского математического портала Math-Net.Ru подразумевает, что вы прочитали и согласны с пользовательским соглашением http://www.mathnet.ru/rus/agreement

Параметры загрузки:

IP : 107.22 .136 .117

26 апреля 2023 г., $18: 31: 18$ 
ТЕОРЕТИЧЕСКАЯ

И МАТЕМАТИЧЕСКАЯ

ФИЗИКА

Том 122, № 2

февраль, 2000

(C) 2000 г.

Г. Фальки*

\section{РЕДУКЦИЯ БИГАМИЛЬТОНОВЫХ СИСТЕМ И РАЗДЕЛЕНИЕ ПЕРЕМЕННЫХ: ПРИМЕР ИЗ ИЕРАРХИИ БУССИНЕСКА}

В общих рамках стационарных потоков $n$-иерархий Гельфанда-Дикого обсуждается система Буссинеска со стационарным временем $t_{5}$. Показано, каким образом аккуратное использование бигамильтоновой структуры позволяет получить множество разделенных координат для соответствующих уравнений Гамильтона-Якоби.

\section{1. ВВЕДЕНИЕ}

Хорошо известно, что уравнение Буссинеска $u_{t t}=(1 / 3)\left(-u_{x x x x}+4 u_{x}^{2}+4 u u_{x x}\right)$, представленное как система уравнений первого порядка в переменных $(u, v)$

$$
\begin{aligned}
& u_{t_{2}}=-u_{x x}+2 v_{x}, \\
& v_{t_{2}}=v_{x x}-\frac{2}{3} u_{x x x}+\frac{2}{3} u u_{x},
\end{aligned}
$$

представляет собой член бесконечной иерархии эволюционных уравнений. Эти уравнения определяются следующим образом. На пространстве $\mathcal{M}_{3}$ дифференциальных операторов третьего порядка $L=\partial_{x}^{3}+u(x) \partial_{x}+v(x)$ мы рассматриваем оператор $L^{1 / 3}$, т.е. псевдодифференшиальный оператор вида

$$
L^{\frac{1}{3}}=\partial+\sum_{i=1}^{\infty} q_{i}(u, v) \partial^{-i}
$$

удовлетворяющий условию $\left(L^{1 / 3}\right)^{3}=L$. Степени оператора $L^{1 / 3}$ определяют бесконечное семейство коммутативных потоков на $\mathcal{M}_{3}$ как

$$
\frac{\partial}{\partial t_{p}} L=\left[L,\left(L^{\frac{p}{3}}\right)_{+}\right]
$$

\footnotetext{
${ }^{*}$ SISSA, Trieste, Italy. E-mail: falqui@sissa.it

†Dipartimento di Matematica, Università di Milano, Milano, Italy.

E-mail: magri@vmimat.mat.unimi.it

‡Dipartimento di Scienze Matematiche, Università di Trieste, Trieste, Italy.

E-mail: tondo@univ.trieste.it
} 
где $(\cdot)_{+}-$проекция на чисто дифференциальную часть псевдодифференциального оператора. Стационарные потоки для такой системы соответствуют подпространству операторов $L$, удовлетворяюших (нелинейному) соотношению $\left[L, L^{p / 3}\right]=0$ для некоторых $p$. В настоящей работе мы обсуждаем случай $p=5$. Соответствующие уравнения для иерархии имеют вид

$$
\begin{aligned}
u_{t_{5}}= & -\frac{1}{9} u_{x x x x x}+\frac{5}{9} u_{x} u_{x x}+\frac{5}{9} u u_{x x x}+\frac{5}{3} u_{x x} v+ \\
& +\frac{5}{3} u_{x} v_{x}-\frac{5}{9} u^{2} u_{x}-\frac{10}{3} v v_{x} \\
v_{t_{5}}= & -\frac{1}{9} v_{x x x x x}+\frac{10}{9} u_{x x x} v+\frac{20}{9} u_{x x} v_{x}+\frac{5}{3} u_{x} v_{x x}+ \\
& +\frac{5}{9} u v_{x x x}-\frac{5}{3} v_{x}^{2}-\frac{5}{3} v v_{x x}-\frac{10}{9} u v u_{x}-\frac{5}{9} u^{2} v_{x}
\end{aligned}
$$

Легко видеть, что пространство $\mathcal{M}_{3}^{(5)}$ нулей поля $\partial / \partial t_{5}$ можно параметризовать данными Коши для двух обыкновенных дифференциальных уравнений пятого порядка от двух переменных. На таком 10-мерном многообразии рассмотрим ограничения первых четырех нетривиальных потоков иерархии Буссинеска

$$
\frac{\partial}{\partial t_{p}} L=\left[L,\left(L^{\frac{p}{3}}\right)_{+}\right], \quad p=1,2,4,7 .
$$

Наша цель состоит в том, чтобы изучить бигамильтонову структуру этих уравнений и показать, как аккуратное использование таких структур позволяет явным образом получить координаты, в которых соответствуюшие уравнения Гамильтона-Якоби разделяются.

В разделах 2 и 3 мы показываем, что на $\mathcal{M}_{3}^{(5)}$ существует линейный пучок скобок Пуассона $\{F, G\}_{\lambda}=\{F, G\}_{1}-\lambda\{F, G\}_{0}$, обладающий следующими свойствами.

1. Они имеют две функции Казимира $H(\lambda)=H_{1} \lambda+H_{2}$ и $K(\lambda)=H_{3} \lambda^{3}+H_{4} \lambda^{2}+$ $H_{5} \lambda+H_{6}$; функции $H_{1}$ и $H_{3}$ представляют собой функции Казимира скобки $\{F, G\}_{0}$, а функции $H_{2}$ и $H_{6}-$ функции Казимира скобки $\{F, G\}_{1}$.

2. Функции $H_{2}, H_{4}, H_{5}$ и $H_{6}$ представляют собой гамильтоновы функции по отношению к скобкам Пуассона $\{F, G\}_{0}$ для четырех рассматриваемых векторных полей, а функции $H_{1}, H_{3}, H_{4}, H_{5}$ - их гамильтоновы функции относительно скобки Пуассона $\{F, G\}_{1}$.

3. Все эти функции находятся в инволюции по отношению ко всему пуассонову пучку.

Мы докажем, что бигамильтонова структура векторных полей (1.5) может быть использована для интегрирования уравнений движения в соответствии со следующей двухшаговой схемой.

Шаг 1. Рассмотрим поверхность уровня $S_{0}$ функций $H_{1}$ и $H_{3}$. Это - 8-мерный симплектический лист скобки Пуассона $\{F, G\}_{0}$. Данные векторные поля являются касательными к $S_{0}$, следовательно их можно ограничить на данное многообразие. Заметим, что обе скобки Пуассона $\{F, G\}_{0}$ и $\{F, G\}_{1}$ ограничиваются на $S_{0}$ с помошью редукции Марсдена-Ратиу. Отсюда можно заключить, что $S_{0}$ наследует структуру Пуассона-Нийенхейса, т.е. $S_{0}$ представляет собой многообразие с симплектической формой $\omega$ 
(индуцированной скобкой $\{F, G\}_{0}$ ) и с согласованным тензором Нийенхейса $N$ (индуцированным скобкой $\left.\{F, G\}_{1}\right)$.

Шаг 2. Построим множество разделенных переменных, определенных на $S_{0}$. Для этого мы используем координаты, введенные в работе [1] и названные координатами Дарбу-Нийенхейса. Они использовались авторами работы [2] как разделяюшиеся переменные для систем типа систем Хенона-Хейлеса, полученных из стационарных потоков иерархии Кортевега-де Фриза (Кд $\Phi)$. В настоящей работе мы явным образом вычисляем такие координаты для стационарных потоков Буссинеска и показываем, что они являются разделяюшимися переменными для уравнений Гамильтона-Якоби, ассоциированных с гамильтоновыми функшиями $H_{2}, H_{4}, H_{5}, H_{6}$.

На наш взгляд, эти потоки являются хорошим примером тесной связи между разделяюшимися координатами и бигамильтоновой структурой солитонных уравнений. Мы выбрали для детального изучения именно эту задачу с целью показать, что такие методы могут эффективно применяться в более сложных, чем уравнение Кд $\Phi$ (см., например, [3-6]), случаях, относящихся к классу бигамильтоновых многообразий максимального ранга, обсуждавшихся в работе [7]. В соответствии с этим мы приведем вычисления вместо того, чтобы доказьвать общие утверждения. Однако мы хотели бы заметить, что данные результаты не специфичны для уравнений Буссинеска. Действительно, как будет ясно из наших аргументов, они выполнены подобным же образом, mutatis mutandis, и для широкого класса стационарных потоков $n$-иерархий Гельфанда-Дикого. Подобная формализация будет рассмотрена в нашей последующей публикации.

\section{2. ЦЕНТРАЛЬНАЯ СИСТЕМА И ЕЕ РЕДУКЦИЯ}

Чтобы получить пары Лакса и гамильтоновы структуры для $t_{5}$-стационарной системы Буссинеска (Bsq5), напомним формализм Кадомцева-Петвиашвили (KП), обсуждавшийся в работах $[8,9]$.

Рассмотрим пространство $\mathcal{M}$ последовательностей рядов Лорана $\left\{H^{(k)}\right\}_{k=1, \ldots, \infty}$ (токов), имеюших вид

$$
H^{(0)}=1, \quad H^{(k)}=z^{k}+\sum_{l \geqslant 1} H_{l}^{k} z^{-l},
$$

где $H_{l}^{k}$ - скаляры. Определим на $\mathcal{M}$ семейство векторных полей следующим образом. Ассоциируем с точкой $\left\{H^{(k)}\right\}_{k=0, \ldots, \infty}$ в $\mathcal{M}$ линейную оболочку $\mathcal{H}_{+}$токов $H^{(k)}$

$$
\mathcal{H}_{+}=\left\langle 1, H^{(1)}, H^{(2)}, \ldots\right\rangle,
$$

являюшуюся подпространством в векторном пространстве $\mathcal{H}$ рядов Лорана от формальной переменной $z$. Далее рассмотрим соотношения инвариантности

$$
\left(\frac{\partial}{\partial t_{j}}+H^{(j)}\right) \mathcal{H}_{+} \subset \mathcal{H}_{+},
$$

как определяющие уравнения для $j$-го векторного поля семейства. В явном виде имеем уравнения

$$
\frac{\partial H^{(k)}}{\partial t_{j}}=H^{(j+k)}-H^{(j)} H^{(k)}+\sum_{l=1}^{k} H_{l}^{j} H^{(k-l)}+\sum_{l=1}^{j} H_{l}^{k} H^{(j-l)},
$$


которые будем в дальнейшем назьвать центральной системой (ЦС). Она имеет следующие свойства:

коммутативность:

$$
\left[\frac{\partial}{\partial t_{j}}, \frac{\partial}{\partial t_{k}}\right]=0
$$

"точность":

$$
\frac{\partial}{\partial t_{k}} H^{(j)}=\frac{\partial}{\partial t_{j}} H^{(k)} \text {. }
$$

Связь ЦС с обычной формулировкой КП-теории (см., например, $[10,11]$ и ссылки в этих работах) как системы эволюционных уравнений Лакса для псевдодифференциальных операторов Сато

$$
\mathcal{Q}=\partial+\sum_{i=1}^{\infty} q_{i} \partial^{-i}
$$

а также доказательство двух указанных выше свойств можно найти в работе [8]. Здесь мы просто напомним, что редукции КП-иерархии к $n$-иерархиям Гельфанда-Дикого получаются путем наложения условия

$$
H^{(n)} \equiv z^{n}
$$

эквивалентного обычной связи $\left(\mathcal{Q}^{n}\right)_{-}=0$. В частности, иерархия Буссинеска соответствует случаю $n=3$.

Необходимо заметить, что на подмножестве $\mathcal{S}_{n}$ из множества $\mathcal{M}$, образованном точками, удовлетворяюшими условию (2.5), $n$-е уравнение ЦС можно рассматривать как уравнение связи

$$
H^{(j+n)}=z^{n} H^{(j)}-\sum_{l=1}^{n} H_{l}^{j} H^{(n-l)},
$$

из которого можно получить рекуррентные формулы для определения коэффициентов Лорана $H^{(k)}, k>n$, в терминах коэффициентов первых $n$ токов. Более формально, имеет место

ПрЕДЛОЖЕНИЕ 1. Подмногообразие $\mathcal{S}_{n}$ является подмножеством в $\mathcal{H}_{+}$, определяемым уравнением

$$
z^{n}\left(\mathcal{H}_{+}\right) \subset \mathcal{H}_{+},
$$

т.е. множеством точек, в которых оператор умножения на $\lambda=z^{n}$ переводит пространство $\mathcal{H}_{+}$в себя. Следовательно, $\mathcal{S}_{n}$ порождается первыми п токами

$$
H^{(0)} \equiv 1, H^{(1)}, \ldots, H^{(n-1)}
$$

над пространством многочленов от $\lambda$ с коэффичиентами в множестве

$$
\left\{H_{l_{1}}^{1}, H_{l_{2}}^{2}, \ldots, H_{l_{n-1}}^{n-1}\right\}, \quad l_{i} \in \mathbb{N} .
$$

Редуиированные уравнения движения $C S_{n}$ представляют собой ограничение ЦС на $\mathcal{S}_{n}$. 
Векторное поле $X_{1}$

$$
\begin{aligned}
& \dot{h}_{1}=k_{1}-2 h_{2} \\
& \dot{k}_{1}=-h_{3}+h_{1}^{2}-k_{2} \\
& \dot{h}_{2}=-2 h_{3}-h_{1}^{2}+k_{2} \\
& \dot{k}_{2}=-k_{3}+h_{2} h_{1}-h_{4}-h_{1} k_{1} \\
& \dot{h}_{3}=-2 h_{2} h_{1}+k_{3}-2 h_{4} \\
& \dot{k}_{3}=-k_{4}-h_{1} k_{2}-h_{5}-k_{1} h_{2}+h_{1} h_{3} \\
& \dot{h}_{4}=-2 h_{5}-h_{2}^{2}-2 h_{1} h_{3}+k_{4} \\
& \dot{k}_{4}=-h_{1} k_{3}+h_{2} h_{1}{ }^{2}-h_{1} h_{4}+h_{1}{ }^{2} k_{1}-2 k_{1} h_{3}-k_{1} k_{2}-2 k_{2} h_{2} \\
& \dot{h}_{5}=3 k_{5}-2 k_{1} k_{2}-2 k_{2} h_{2}-2 h_{2} h_{3}-2 k_{1} h_{3}+2 h_{2} h_{1}^{2}+2 h_{1}^{2} k_{1}-6 h_{1} h_{4} \\
& \dot{k}_{5}=-3 k_{2} h_{3}-k_{1} h_{4}-h_{1} k_{1}{ }^{2}-h_{2} k_{3}-h_{2} h_{4}+h_{2}{ }^{2} h_{1}-k_{1} k_{3}
\end{aligned}
$$

\section{Векторное поле $X_{2}$}

$$
\begin{array}{|l}
\dot{h}_{1}=-h_{3}+h_{1}^{2}-k_{2} \\
\dot{k}_{1}=h_{4}+h_{1} k_{1}-h_{2} h_{1}-2 k_{3} \\
\dot{h}_{2}=-k_{3}+h_{2} h_{1}-h_{4}-h_{1} k_{1} \\
\dot{k}_{2}=-k_{1}^{2}-h_{2}^{2}+h_{5}-h_{1} k_{2}-2 k_{4}+2 k_{1} h_{2} \\
\dot{h}_{3}=-k_{4}-h_{1} k_{2}-h_{5}-k_{1} h_{2}+h_{1} h_{3} \\
\dot{k}_{3}=-3 k_{5}-k_{1} k_{2}+k_{2} h_{2}-h_{2} h_{1}^{2}-h_{1} k_{3}+2 h_{1} h_{4}-h_{1}^{2} k_{1}+3 k_{1} h_{3}-h_{2} h_{3} \\
\dot{h}_{4}=-h_{1} k_{3}+h_{2} h_{1}^{2}-h_{1} h_{4}+h_{1}^{2} k_{1}-2 k_{1} h_{3}-k_{1} k_{2}-2 k_{2} h_{2} \\
\dot{k}_{4}=h_{1} k_{1}^{2}-h_{2}^{2} h_{1}+h_{1} h_{5}-2 h_{1} k_{4}+2 k_{1} h_{4}-4 k_{1} k_{3}-k_{2} h_{3}-k_{2}{ }^{2} \\
\dot{h}_{5}=-3 k_{2} h_{3}-k_{1} h_{4}-h_{1} k_{1}^{2}-h_{2} k_{3}-h_{2} h_{4}+h_{2}^{2} h_{1}-k_{1} k_{3} \\
\dot{k}_{5}=h_{2} k_{1}{ }^{2}-h_{2}{ }^{3}+h_{2} h_{5}-h_{2} h_{1} k_{2}-2 h_{2} k_{4}+k_{1} h_{2}^{2}-k_{1}{ }^{3}+k_{1} h_{5}- \\
\quad-h_{1} k_{1} k_{2}-2 k_{1} k_{4}-3 k_{3} k_{2}
\end{array}
$$

Таким образом, в случае $n=3$ в соответствии с предложением 1 степени свободы можно собрать в два набора:

$$
H^{(1)} \equiv h=z+\sum_{i=1}^{\infty} h_{i} z^{-i}, \quad H^{(2)} \equiv k=z^{2}+\sum_{i=1}^{\infty} k_{i} z^{-i} .
$$

Мы пока все еще имеем динамическую систему с бесконечным числом степеней свободы. Чтобы получить конечномерные динамические системы, представляющие ограничение потоков на стационарные многообразия, можно заметить, что множество $\mathcal{Z}_{p}$ нулей $р$-го 
векторного поля также является инвариантным подмногообразием для уравнений (2.4), так что можно ограничить потоки на пересечения инвариантных многообразий $\mathcal{S}_{n} \cap \mathcal{Z}_{p}$. В частности, для изучения Bsq5-системы мы рассмотрим пространство $\mathcal{M}_{3}^{(5)}=\mathcal{S}_{3} \cap$ $\mathcal{Z}_{5}$. Для построения ограниченных потоков заметим, что коэффициенты в разложении Лорана уравнений

$$
\frac{\partial}{\partial t_{5}} h=0, \quad \frac{\partial}{\partial t_{5}} k=0
$$

дают полиномиальные уравнения для коэффициентов $\left(h_{i}, k_{j}\right), i, j \geqslant 6$, которые можно решить рекуррентным способом относительно первых десяти переменных $\left\{h_{i}, k_{i}\right.$, $i=1, \ldots, 5\}$. В соответствии с этим после такой подстановки получим систему полиномиальных векторных полей $X_{j}$, определенную на фазовом пространстве $\mathcal{M}_{3}^{(5)}$. Первые члены этой системы представлены в табл. 1 и 2.

\section{3. ПРЕДСТАВЛЕНИЕ ЛАКСА И СТРУКТУРЫ ПУАССОНА}

Другое следствие из предложения 1 в случае $n=3$ заключается в том, что для каждого $j \neq 3 l$ сушествует нетривиальная $(3 \times 3)$-матрица $\mathcal{V}^{(j)}(\lambda)$, полиномиальньм образом зависящая от $\lambda$ и такая, что

$$
\left(\frac{\partial}{\partial t_{j}}+H^{(j)}\right)\left[\begin{array}{l}
1 \\
h \\
k
\end{array}\right]=\mathcal{V}^{(j)}(\lambda)\left[\begin{array}{l}
1 \\
h \\
k
\end{array}\right]
$$

Свойства коммутативности и "точности" ЦС приводят к представлению нулевой кривизны (или Захарова-Шабата) для ограничения ЦС на $\mathcal{S}_{3}$ :

$$
\frac{\partial}{\partial t_{j}} \mathcal{V}^{(k)}(\lambda)-\frac{\partial}{\partial t_{k}} \mathcal{V}^{(j)}(\lambda)+\left[\mathcal{V}^{(k)}(\lambda), \mathcal{V}^{(j)}(\lambda)\right]=0
$$

Теперь легко получить задачу Лакса для Bsq5-системы на $\mathcal{M}_{3}^{(5)}$. Действительно, на стационарном многообразии поля $X_{5}=\partial / \partial t_{5}$ мы немедленно получаем из уравнений $(3.2)$, что матрица Лакса $\mathcal{V}^{(5)}(\lambda)$ удовлетворяет уравнениям

$$
\frac{\partial}{\partial t_{k}} \mathcal{V}^{(5)}(\lambda)=\left[\mathcal{V}^{(k)}(\lambda), \mathcal{V}^{(5)}(\lambda)\right]
$$

Другими словами, на матрице $\mathcal{V}^{(5)}$ все потоки из $\mathcal{M}_{3}^{(5)}$ являются эволюционными уравнениями Лакса. Матрица Лакса $\mathcal{V}^{(5)}$ (вычисленная с использованием уравнения (2.6)) имеет следующий явный вид:

$$
\begin{aligned}
\mathcal{V}^{(5)}(\lambda)=\lambda^{2} & {\left[\begin{array}{lll}
0 & 0 & 0 \\
1 & 0 & 0 \\
0 & 1 & 0
\end{array}\right]+\lambda\left[\begin{array}{ccc}
0 & 0 & 1 \\
h_{2} & h_{1} & 0 \\
-h_{3}+k_{2} & k_{1}-h_{2} & -h_{1}
\end{array}\right]+} \\
+ & {\left[\begin{array}{ccc}
-k_{3} & -k_{2} & -k_{1} \\
L_{0}^{2,1} & -h_{2} h_{1}+h_{4} & -h_{1}^{2}+h_{3} \\
L_{0}^{3,1} & L_{0}^{3,2} & h_{2} h_{1}-h_{4}+k_{3}
\end{array}\right] }
\end{aligned}
$$


где

$$
\begin{aligned}
L_{0}^{2,1}= & -h_{1} k_{2}+k_{4}-k_{1}{ }^{2}-h_{1} h_{3}+h_{5}, \\
L_{0}^{3,2}= & -k_{1} h_{2}+h_{2}{ }^{2}-h_{5}+2 k_{4}-k_{1}{ }^{2}, \\
L_{0}^{3,1}= & h_{1} k_{3}-2 h_{1} h_{4}+3 k_{5}+h_{2} h_{1}{ }^{2}-2 k_{1} k_{2} \\
& -2 k_{1} h_{3}+h_{1}{ }^{2} k_{1}-2 k_{2} h_{2}+h_{2} h_{3} .
\end{aligned}
$$

Можно заметить, что отображение $\left\{h_{1}, \ldots, k_{5}\right\} \mapsto \mathcal{V}^{(5)}$ является обратимым, и, следовательно, уравнения Лакса (3.3) точно представляют Bsq5-систему.

Вторые члены пары Лакса для потоков $X_{1}$ и $X_{2}$ задаются уравнениями

$$
\mathcal{V}^{(1)}=\left[\begin{array}{ccc}
0 & 1 & 0 \\
2 h_{1} & 0 & 1 \\
\lambda+h_{2}+k_{1} & h_{1} & 0
\end{array}\right], \quad \mathcal{V}^{(2)}=\left[\begin{array}{ccc}
0 & 0 & 1 \\
\lambda+h_{2}+k_{1} & h_{1} & 0 \\
-h_{3}+2 k_{2} & \lambda-h_{2}+2 k_{1} & -h_{1}
\end{array}\right]
$$

3.1. Гамильтоновы структуры. Важное свойство векторных полей $\mathrm{Bsq}_{5}$-системы состоит в том, что, кроме представления Лакса, они допускают бигамильтоново представление или формулировку Пуассона $c$ параметром. Как мы видели, формулировка задачи Лакса происходит из ЦС. Пуассонову формулировку можно получить следуюшим образом.

Хорошо известно [12-14], что на пространстве $\mathfrak{g}[[\lambda]]$ лорановских полиномов со значениями в алгебре Ли $\mathfrak{g}$ имеется семейство взаимно согласованных пуассоновых тензоров $\mathcal{P}_{l}$, связанных с семейством классических $R$-матриш

$$
R_{l}(X(\lambda))=\left(\lambda^{l} X(\lambda)\right)_{+}-\left(\lambda^{l} X(\lambda)\right)_{-} .
$$

Некоторые из них (включая соответствуюшие значениям $l=0,1$ ) ограничиваются на аффинное многообразие

$$
\mathfrak{g}_{n}^{A}:=\left\{X \in \mathfrak{g}[[\lambda]] \mid X(\lambda)=\lambda^{n} A+\sum_{i=0}^{n-1} \lambda^{i} X_{i}\right\},
$$

где $A$ - фиксированный элемент алгебры g.

Возможно, менее известным является факт, на который указывалось в работе [15], что $\mathcal{P}_{0}$ и $\mathcal{P}_{1}$ допускают дальнейшие редукции, ведушие к обобщенным мамфордовским системам. Мы хотим показать, что обсуждавшиеся в разделе 2 гамильтоновы структуры для Bsq5-системы, получаемые путем редукции ЦС, являются бигамильтоновой редукцией Марсдена-Ратиу $(\mathrm{MP})$ пары $\mathcal{P}_{0}$ и $\mathcal{P}_{1}$. Такой выбор пуассоновой пары является отнюдь не случайным. Действительно, из бигамильтонова уравнения на $\mathfrak{g}_{n}^{A}$

$$
\dot{X}=\left(\mathcal{P}_{1}-\lambda \mathcal{P}_{0}\right) \nabla F
$$

следует, что полином $X(\lambda)$ эволюционирует в соответствии с уравнением Лакса.

Напомним, что теорема о МР-редукции [16] рассматривает многообразие Пуассона $(\mathcal{M}, P)$, подмногообразие $\mathcal{S} \hookrightarrow \mathcal{M}$ и обобшенное пространство векторных полей ("распределение") $\left.D \subset T \mathcal{M}\right|_{\mathcal{S}}$, такие что $E=D \cap T \mathcal{S}$ представляет собой регулярное слоение с хорошим фактором $\mathcal{N}=\mathcal{S} / E$. Теорема утверждает, что пуассонов тензор $P$ редуцируется к $\mathcal{N}$, если выполнены следуюшие условия: 
1) функции на $M$, инвариантные вдоль "распределения" $D$, образуют пуассонову подалгебру в $C^{\infty}(\mathcal{M})$;

2) $P\left(D^{0}\right) \subset T \mathcal{S}+D$, где $D^{0}$ - аннулятор $D$ в $T^{*} \mathcal{M}$.

Редуцированные пуассоновы тензоры можно вычислить согласно следуюшей схеме:

Выберем ковектор $v_{n}^{\mathcal{N}} \in T_{n}^{*} \mathcal{N}$.

Выберем точку $s \in \mathcal{S}$ в слое над $n$ и поднимем $v_{n}^{\mathcal{N}}$ до ковектора $v_{s}^{\mathcal{M}} \in T_{s}^{*} \mathcal{M}$, т.е. в продолжение $\pi^{*} v_{n}^{\mathcal{N}}$, лежащее в аннуляторе $D^{0}$ "распределения" $D$.

Построим векторное поле $\left(P^{\mathcal{M}}\right)_{s} v_{s}^{\mathcal{M}}$, ассоциированное с поднятым ковектором $v_{s}^{\mathcal{M}}$ посредством пуассонова тензора $\mathcal{M}$ в точке $s$. Теорема о МР-редукции утверждает, что вектор $\left(P^{\mathcal{M}}\right)_{s} v_{s}^{\mathcal{M}}$ проецируется на $\mathcal{N}$. Проекция не зависит ни от специального выбора расширения $v_{s}^{\mathcal{M}}$, ни от точки $s$ на кривой и определяет $\left(P^{\mathcal{N}}\right)_{n} v_{n}^{\mathcal{N}}$.

Теорема о бигамильтоновой МР-редукиии [17] рассматривает многообразие $\left(\mathcal{M}, P_{0}, P_{1}\right)$, снабженное парой согласованных пуассоновых структур, т.е. бигамильтоново многообразие. Она является следствием теоремы о МР-редукции, вытекаюшим из того, что бигамильтоново многообразие допускает некоторый тип двойного слоения. Это подсказывает выбор $\mathcal{S}$ как симплектического листа пуассонова тензора $P_{0}$ и $D=P_{1}\left(\operatorname{Ker} P_{0}\right)$. Если $\mathcal{S}$ выбирается так, что предположение регулярности теоремы о МР-редукции выполнено, то упомянутые выше условия 1 и 2 являются следствием совместности пуассоновых пар $\left(P_{0}, P_{1}\right)$. Более того, теорема утверждает факт приводимости всего пуассонова пучка $P_{\lambda}=P_{1}-\lambda P_{0}$ и снабжает $\mathcal{N}$ бигамильтоновой структурой, которую можно вычислить в соответствии с очерченной вьше процедурой.

Рассмотрим в нашей Bsq5-задаче редукцию пуассонова пучка $\mathcal{P}_{\lambda}=\mathcal{P}_{1}-\lambda \mathcal{P}_{0}$, ассоциированного с $R$-матрицами (3.7), который определен на пространстве бесследовых $(3 \times 3)$-матриц вида $X(\lambda)=\lambda^{2} A+\lambda x_{1}+x_{0}$, где

$$
A=\left[\begin{array}{lll}
0 & 0 & 0 \\
1 & 0 & 0 \\
0 & 1 & 0
\end{array}\right] .
$$

Используя форму Киллинга на $\mathfrak{s l}(3)$ и вычет по $\lambda$, можно параметризовать касательные и кокасательные векторы как матрично-значные (лорановские) полиномы:

$$
\dot{X}=\lambda \dot{x}_{1}+\dot{x}_{0}, \quad W=\frac{w_{0}}{\lambda}+\frac{w_{1}}{\lambda^{2}} .
$$

Пуассоновы тензоры $\mathcal{P}_{0}$ и $\mathcal{P}_{1}$ имеют следуюший явный вид [14]:

$$
\begin{aligned}
& \dot{X}=\mathcal{P}_{0}(W) \Leftrightarrow\left(\begin{array}{c}
\dot{x}_{0} \\
\dot{x}_{1}
\end{array}\right)=\left(\begin{array}{cc}
{\left[x_{1}, \cdot\right]} & {[A, \cdot]} \\
{[A, \cdot]} & 0
\end{array}\right)\left(\begin{array}{l}
w_{0} \\
w_{1}
\end{array}\right)=\left(\begin{array}{c}
{\left[x_{1}, w_{0}\right]+\left[A, w_{1}\right]} \\
{\left[A, w_{0}\right]}
\end{array}\right), \\
& \dot{X}=\mathcal{P}_{1}(W) \Leftrightarrow\left(\begin{array}{c}
\dot{x}_{0} \\
\dot{x}_{1}
\end{array}\right)=\left(\begin{array}{cc}
-\left[x_{0}, \cdot\right] & 0 \\
0 & {[A, \cdot]}
\end{array}\right)\left(\begin{array}{l}
w_{0} \\
w_{1}
\end{array}\right)=\left(\begin{array}{c}
{\left[w_{0}, x_{0}\right]} \\
{\left[A, w_{1}\right]}
\end{array}\right) .
\end{aligned}
$$

Прямые вычисления показывают, что матриша Лакса $\mathcal{V}^{(5)}$ вписывается в предложенную здесь схему, так что посредством бигамильтоновой МР-редукции пространство $\mathcal{M}_{3}^{(5)}$ снабжается пуассоновым пучком $P_{\lambda}=P_{1}-\lambda P_{0}$; в координатах $\left(h_{1}, k_{1}, h_{2}, k_{2}, \ldots, h_{5}, k_{5}\right)$ тензоры $P_{0}$ и $P_{1}$ имеют вид, представленный в табл. 3 и 4. 
Таблица 3

Редуцированный тензор $P_{0}$

\begin{tabular}{|cccccccccc|}
\hline 0 & 0 & 0 & 0 & 0 & 0 & 0 & 0 & -1 & 0 \\
& 0 & 0 & 0 & 0 & 1 & 0 & 0 & 0 & 0 \\
& & 0 & 0 & 0 & 1 & 1 & 0 & 0 & 0 \\
& & & 0 & 1 & 0 & 0 & 0 & 0 & 0 \\
& & & & 0 & 0 & 0 & $-h_{1}$ & $-2 h_{1}$ & $-k_{1}-h_{2}$ \\
& & & & 0 & $-h_{1}$ & $-2 k_{1}$ & $-k_{1}-h_{2}$ & $-2 k_{2}$ \\
& & & & & 0 & 0 & $-2 h_{2}$ & $-k_{2}$ \\
& 0 & & & & & 0 & $-k_{2}$ & 0 \\
& & & & & & 0 & 0 \\
& & & & & & & 0 & 0 \\
\hline
\end{tabular}

Таблица 4

Редуцированный тензор $P_{1}$.

$\frac{1}{3} \times$\begin{tabular}{cccccccccc|}
0 & 0 & -3 & 0 & 0 & 0 & $-3 h_{1}$ & $2 h_{2}-k_{1}$ & $k_{1}-2 h_{2}$ & $P^{1,10}$ \\
& 0 & 0 & 0 & 0 & $3 k_{1}$ & 0 & $h_{3}-h_{1}{ }^{2}+k_{2}$ & $2 h_{3}-2 h_{1}{ }^{2}-k_{2}$ & $P^{2,10}$ \\
& & 0 & 0 & $6 h_{1}$ & $3 k_{1}$ & $3 h_{2}$ & $2 k_{2}+2 h_{3}+h_{1}{ }^{2}$ & $k_{2}+4 h_{3}+2 h_{1}{ }^{2}$ & $P^{3,10}$ \\
& & & 0 & $3 k_{1}$ & $3 k_{2}$ & $3 k_{2}$ & $P^{4,8}$ & $P^{4,9}$ & $P^{4,10}$ \\
& & & & 0 & 0 & $-3 h_{1}{ }^{2}-3 h_{3}$ & $P^{5,8}$ & $k_{3}-2 h_{2} h_{1}-2 h_{4}$ & $P^{5,10}$ \\
& & & & 0 & $-3 h_{1} k_{1}$ & $P^{6,8}$ & $P^{6,9}$ & $P^{6,10}$ \\
& & & & & & 0 & $P^{7,8}$ & $P^{7,9}$ & $P^{7,10}$ \\
& & & & & & 0 & $P^{8,9}$ & $P^{8,10}$ \\
& & & & & & & 0 & $P^{9,10}$ \\
& & & & & & & & & 0 \\
\hline
\end{tabular}

В табл. 4 элементы $P^{l, k}$ - многочлены в координатах $\left\{h_{1}, \ldots, k_{5}\right\}$ :

$$
\begin{aligned}
P^{1,10} & =h_{3}-2 k_{2}-h_{1}{ }^{2}, \\
P^{2,10} & =-h_{1} k_{1}+h_{2} h_{1}-h_{4}+2 k_{3}, \\
P^{3,10} & =k_{3}+h_{4}-h_{2} h_{1}+h_{1} k_{1}, \\
P^{4,8} & =k_{3}+h_{4}-h_{2} h_{1}+h_{1} k_{1}, \\
P^{4,9} & =2 k_{3}+2 h_{4}-2 h_{2} h_{1}+2 h_{1} k_{1}, \\
P^{4,10} & =-2 k_{1} h_{2}+k_{1}{ }^{2}+h_{1} k_{2}-h_{5}+h_{2}{ }^{2}+2 k_{4}, \\
P^{5,8} & =-3 h_{1} k_{1}+2 h_{2} h_{1}+2 h_{4}-k_{3}, \\
P^{5,10} & =-h_{1} h_{3}-5 h_{1} k_{2}-2 k_{1} h_{2}-3 k_{1}{ }^{2}+h_{5}+k_{4}, \\
P^{6,8} & =-6 k_{1}^{2}+k_{1} h_{2}-2 h_{1} k_{2}+k_{4}-h_{1} h_{3}+h_{5}, \\
P^{6,9} & =-3 k_{1}^{2}-k_{1} h_{2}-4 h_{1} k_{2}+2 k_{4}-2 h_{1} h_{3}+2 h_{5}, \\
P^{6,10} & =-3 k_{1} h_{3}-8 k_{1} k_{2}-4 k_{2} h_{2}+h_{1} k_{3}+h_{2} h_{3}-2 h_{1} h_{4}+h_{2} h_{1}{ }^{2}+3 k_{5}+h_{1}{ }^{2} k_{1},
\end{aligned}
$$




$$
\begin{aligned}
P^{7,8}= & h_{2}{ }^{2}+2 h_{1} h_{3}-k_{4}+2 h_{5}, \\
P^{7,9}= & -h_{2}{ }^{2}-3 h_{1} k_{2}+4 h_{1} h_{3}-3 k_{1}{ }^{2}+4 k_{4}+h_{5}, \\
P^{7,10}= & h_{1} k_{3}+h_{1} h_{4}-h_{2} h_{1}{ }^{2}-h_{1}{ }^{2} k_{1}-4 k_{2} h_{2}-2 k_{1} k_{2}+2 k_{1} h_{3}, \\
P^{8,9}= & -4 k_{2} h_{2}-4 h_{1} h_{4}+2 k_{1} h_{3}-3 k_{1} k_{2}+3 k_{5}+2 h_{1} k_{3}-2 h_{2} h_{3}, \\
P^{8,10}= & -2 k_{2}{ }^{2}-h_{1} h_{5}+2 h_{1} k_{4}-2 k_{2} h_{3}-3 k_{1} h_{4}-2 h_{1} k_{1}{ }^{2}-h_{2} k_{3}- \\
& -h_{2} h_{4}+2 h_{2}{ }^{2} h_{1}+3 k_{1} k_{3}, \\
P^{9,10}= & -3 k_{2} h_{3}-k_{1} h_{4}-h_{1} k_{1}{ }^{2}-h_{2} k_{3}-h_{2} h_{4}+h_{2}{ }^{2} h_{1}-k_{1} k_{3} .
\end{aligned}
$$

Отметим, что в случае $t_{4}$-стационарной иерархии Буссинеска эта процедура дает пару пуассоновых структур, согласующихся с полученными в работе [18].

В соответствии с теоремой Гельфанда-Захаревича [7] гамильтоновы функции для потоков можно вычислить, если найти функции Казимира для пуассонова пучка $P_{1}-\lambda P_{0}$. Однако, поскольку мы имеем дело с задачей Лакса со спектральным параметром, их можно также получить из коэффищиентов спектральной кривой, ассоциированной с нашей задачей, т.е.

$$
0=\operatorname{det}\left(\mu-\mathcal{V}^{(5)}\right)=\mu^{3}-\mu\left(H_{1} \lambda+H_{2}\right)-\left(\lambda^{5}+H_{3} \lambda^{3}+H_{4} \lambda^{2}+H_{5} \lambda+H_{6}\right) .
$$

Явные выражения для гамильтоновых функций имеют вид

$$
\begin{aligned}
& H_{1}=3 k_{5}-3 k_{1} k_{2}-3 k_{2} h_{2}, \quad H_{3}=-3 h_{1} k_{2}+3 k_{4}-3 k_{1}^{2}, \\
& H_{2}=-3 k_{5} k_{1}-h_{5} k_{2}+h_{3} k_{1}^{2}-2 h_{2} h_{1} h_{4}-h_{4} k_{3}+h_{3} h_{2}^{2}-h_{3} h_{5}- \\
& -h_{1} k_{3} k_{1}+h_{2} h_{1} k_{3}+h_{1} h_{3} k_{2}+h_{1} k_{2}^{2}+3 k_{1}^{2} k_{2}-k_{4} k_{2}+2 k_{2} h_{2} k_{1}+ \\
& +k_{3}^{2}+h_{4}^{2}-2 h_{1}{ }^{2} k_{4}+2 h_{3} k_{4}-2 h_{3} k_{1} h_{2}+2 h_{1} h_{4} k_{1}+h_{1}{ }^{2} h_{5} \text {, } \\
& H_{4}=-2 k_{1} k_{4}-2 k_{2} h_{4}+k_{3} h_{3}-3 h_{1} k_{5}-2 h_{2} h_{5}+3 h_{1} k_{1} k_{2}+ \\
& +4 h_{2} h_{1} k_{2}+h_{2}{ }^{3}+k_{1}{ }^{3}-h_{1} h_{2} h_{3}+h_{2} k_{4}+h_{4} h_{3}-h_{1}{ }^{2} k_{3}+ \\
& +k_{1} h_{5}+h_{1} h_{3} k_{1}-k_{1} h_{2}^{2}+k_{3} k_{2}-h_{2} h_{1}^{3}-h_{1}{ }^{3} k_{1}+2 h_{1}{ }^{2} h_{4}, \\
& H_{5}=h_{1} k_{1}^{2} k_{2}+h_{4} h_{3} k_{1}+3 k_{1} k_{2} h_{4}+2 h_{2} h_{1}^{3} k_{1}+h_{1} h_{5} h_{3}-3 h_{1} k_{2} k_{4}+3 h_{1} k_{1} k_{5}+ \\
& +h_{1} h_{4} k_{3}-2 h_{2} k_{1} k_{4}+k_{3} k_{2} h_{2}-3 h_{2} h_{1}{ }^{2} h_{4}+k_{2} h_{2} h_{4}-2 h_{1} k_{4} h_{3}-2 h_{2}{ }^{2} h_{1} k_{2}- \\
& -3 k_{1} h_{1}{ }^{2} h_{4}+3 h_{2} h_{1} k_{5}+h_{3} k_{1} k_{3}+h_{2}{ }^{2} h_{5}+h_{5} k_{4}-h_{5} k_{1}{ }^{2}+h_{3}{ }^{2} k_{2}+h_{2}{ }^{2} h_{1}{ }^{3}- \\
& -h_{1} k_{3}{ }^{2}-k_{2}{ }^{2} h_{3}+h_{1}{ }^{3} k_{1}{ }^{2}+h_{2} k_{1}{ }^{3}+h_{2}{ }^{2} k_{4}-k_{1} h_{2}{ }^{3}-3 h_{4} k_{5}-4 k_{1}{ }^{2} k_{4}+ \\
& +2 h_{1}{ }^{2}{k_{2}}^{2}+2 h_{1} h_{4}^{2}+k_{1} h_{2} h_{5}-h_{2} h_{3} k_{3}-h_{2} h_{4} h_{3}+2 k_{4}{ }^{2}+2 k_{1}{ }^{4}- \\
& -h_{5}^{2}-5 h_{2} h_{1} k_{1} k_{2} \text {, } \\
& H_{6}=-k_{1}{ }^{2} k_{2} h_{4}-h_{1} k_{1}{ }^{3} k_{2}-3 h_{2} h_{1}{ }^{2} k_{2}{ }^{2}-3 k_{5} k_{2} h_{3}-k_{1}{ }^{2} h_{2} h_{1}{ }^{3}+2 k_{1} k_{2}{ }^{2} h_{3}+ \\
& +h_{1}^{2} k_{1}^{2} k_{3}-k_{2} h_{4} k_{4}+h_{1} k_{2}^{2} h_{4}-h_{5} k_{1} k_{4}+2 k_{2}^{2} h_{2} h_{3}-2 k_{4} h_{1}^{2} k_{3}+ \\
& +k_{1}{ }^{2} h_{2} h_{5}+h_{1}{ }^{4} k_{2} h_{2}-k_{1}{ }^{2} k_{2} k_{3}-2 h_{1} k_{1} h_{4}{ }^{2}+2 k_{1} h_{3}{ }^{2} k_{2}+k_{1}{ }^{2} h_{1}{ }^{2} h_{4}- \\
& -k_{1}{ }^{2} h_{3} k_{3}+k_{4} k_{3} k_{2}+3 h_{4} k_{1} k_{5}+2 k_{4} h_{3} k_{3}-2 h_{4} h_{3} k_{1}{ }^{2}- \\
& -3 h_{2} h_{1} k_{1} k_{5}-h_{1} k_{1} k_{2} h_{5}+h_{2} h_{1} k_{2} h_{5}+2 h_{1} k_{1} k_{2} k_{4}- \\
& -2 k_{1} k_{2} h_{2} h_{4}+3 h_{2}{ }^{2} h_{1} k_{1} k_{2}+h_{2} h_{1} h_{3} k_{1}{ }^{2}-2 h_{2} h_{1} h_{4} k_{3}-h_{2} h_{1}{ }^{2} k_{2} h_{3}+ \\
& +3 h_{2} h_{1}{ }^{2} k_{1} h_{4}-2 h_{1} k_{2} h_{3} k_{3}+2 h_{1} h_{3} k_{1} k_{4}+3 h_{1} k_{2} h_{4} h_{3}-3 h_{1}{ }^{2} k_{2} h_{3} k_{1}- \\
& -k_{1} h_{2}{ }^{2} k_{4}+3 h_{1}{ }^{2} k_{2} k_{5}-k_{2} h_{4} h_{5}+k_{3} k_{2} h_{5}+h_{2} h_{1} k_{3}{ }^{2}+h_{5} h_{1}{ }^{2} k_{3}-
\end{aligned}
$$




$$
\begin{aligned}
& -h_{5} h_{3} k_{3}+h_{2}{ }^{2} h_{3} k_{3}-h_{2} h_{3}{ }^{2} k_{2}-k_{1} h_{2}{ }^{2} h_{5}-k_{1} h_{2}{ }^{2} h_{1}{ }^{3}-h_{1} k_{2}{ }^{2} k_{3}+h_{1}{ }^{4} k_{2} k_{1}- \\
& -2 h_{1}{ }^{3} k_{2} h_{4}+k_{1}{ }^{2} h_{2} k_{4}-h_{1} h_{3} k_{1}{ }^{3}+h_{1}{ }^{3} k_{2} k_{3}- \\
& -2 h_{1}{ }^{2} k_{2}{ }^{2} k_{1}+3 k_{1}{ }^{3} k_{4}+h_{4}{ }^{2} k_{3}-h_{4} k_{3}{ }^{2}+k_{1} h_{5}{ }^{2}- \\
& -2 k_{1} k_{4}{ }^{2}+k_{1}{ }^{3} h_{2}{ }^{2}-k_{1}{ }^{4} h_{2}+h_{2} h_{1} k_{2} k_{4}-k_{1} h_{2} h_{3} k_{3}- \\
& -h_{1} k_{1} h_{5} h_{3}+h_{1} h_{4} k_{1} k_{3}+k_{1} h_{2} h_{4} h_{3}-k_{1}{ }^{5} .
\end{aligned}
$$

Прямые вычисления показывают, что имеет место

ПРЕДЛОЖЕНИЕ 2. Коэффичиенты $H_{j}$ характеристического многочлена матрииы Лакса $\mathcal{V}^{(5)}(\lambda)$, векторных полей $\left(X_{1}, X_{2}, X_{4}, X_{7}\right)$ и пуассоновых тензоров $P_{0} u$ $P_{1}$ из табл. 3 и 4 удовлетворяют бигамильтоновым последовательностям, показанным на рисунке. В частности, гамильтоновы функиии $H_{j}$ находятся во взаимной инволющии по отношению ко всему пуассонову пучку.

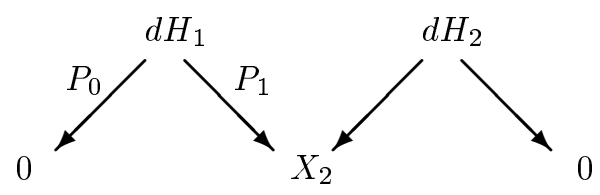

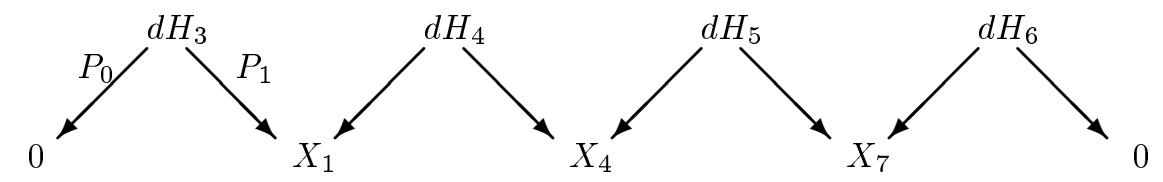

Бигамильтоновы последовательности

\section{4. МНОГООБРАЗИЯ ПУАССОНА-НИЙЕНХЕЙСА И ПРОЦЕДУРА СКЛЯНИНА}

В этом разделе мы покажем, как бигамильтонову структуру Bsq5-иерархии можно использовать, для того чтобы получить множество переменных, удовлетворяющих уравнениям Склянина для разделения переменных. Такой тип переменных возникает в действительности как множество координат, которые придают "канонический” вид структуре Пуассона-Нийенхейса (ПН), получающейся дальнейшей МР-редукцией пуассонова пучка $P_{\lambda}$.

4.1. Редукция пуассонова пучка. Пусть $M$ - бигамильтоново многообразие с вырожденными пуассоновыми тензорами $\left(P_{0}, P_{1}\right)$, и пусть $X_{j}$ - векторное поле бигамильтоновой последовательности на $M$. Возможный способ проанализировать интегрируемость поля $X_{j}$ состоит в том, чтобы исключить функции Казимира одного из пуассоновых тензоров, например $P_{0}$, путем фиксирования значений его функций Казимира. Конечно, $P_{0}$ можно ограничить на любом из его симплектических листов $S_{0}$, а поля $X_{j}$, являюшиеся касательными к $S_{0}$, также можно ограничить до векторных полей $\widehat{X}_{j}$. Эти поля все еше гамильтоновы на $S_{0}$, при этом гамильтонианы $\widehat{H}_{j}$ получаются путем 
ограничений исходных гамильтонианов на $S_{0}$. Однако, вообще говоря, бигамильтонова формулировка теряется при ограничении на $S_{0}$.

Тем не менее в нашей ситуации специфические особенности пучка позволяют редуцировать бигамильтоновы структуры на любой лист $S_{0}$ пуассонова тензора $P_{0}$ общего положения. Действительно, пуассонов пучок $P_{\lambda}$ индуцирует на $S_{0}$ ПН-структуру $[12$, 19]. По отношению к такой ПН-структуре поле $\widehat{X}_{j}$ допускает формулировку, которая будет введена в разделе 5 , достаточную, чтобы позволить найти множество разделяюшихся переменных.

Лемма. Пусть $\widehat{\mathcal{D}}$ - “распределение”, порожденное векторныци полями

$$
Z_{1}=\frac{\partial}{\partial k_{5}}, \quad Z_{2}=\frac{\partial}{\partial k_{4}}+2 \frac{\partial}{\partial h_{5}}
$$

Тогда

$$
L_{\phi_{1} Z_{1}+\phi_{2} Z_{2}}\left(P_{1}-\lambda P_{0}\right)=Z_{1} \wedge W_{1}+Z_{2} \wedge W_{2}
$$

где $L_{X} P$ - производная Ли тензора $P$ вдоль $X, \phi_{1}$ и $\phi_{2}$ - гладкие функиии общего положения, $a W_{i}-$ подходящие векторные поля (зависящие от $\phi_{1}$ и $\left.\phi_{2}\right)$.

Такое условие гарантирует, что пространство функций, аннулируемых "распределением" $\widehat{\mathcal{D}}$, в действительности является пуассоновой подалгеброй в $C^{\infty}\left(\mathcal{M}_{3}^{(5)}\right)$. Более того, "распределение" $\widehat{\mathcal{D}}$ является, вообше говоря, трансверсальнымм к образу тензора $P_{0}$. Следовательно, мы можем применить теорему о МР-редукции и получить следующее

ПРедЛОЖЕНИЕ 3. Пучок $P_{\lambda}$ является редуцируемым $\kappa$ пучку $\widehat{P}_{\lambda}$ на $S_{0}$. Поскольку $\widehat{P}_{0}$ обратим на $S_{0}$, т.е. $\omega=\widehat{P}_{0}^{-1}$ является симплектической 2-формой, $S_{0}$ допускает структуру ПН-многообразия относительно парь $\left(\widehat{P}_{0}, N=\widehat{P}_{1} \widehat{P}_{0}^{-1}\right)$.

Удобный выбор координат на $\mathcal{M}$, соответствуюший "распределению" $\widehat{\mathcal{D}}$, имеет вид $\left\{h_{1}, \ldots, h_{4}, g_{1}=2 k_{4}-h_{5}, v_{1}=H_{1}, v_{2}=H_{3}\right\}$. Таким образом, можно получить редуцированные пуассоновы тензоры из тензоров $P_{0}$ и $P_{1}$ (записанных в новой координатной системе), просто удаляя по два последних ряда и столбца. Их можно представить в виде

$$
\widehat{P}_{0}=\left[\begin{array}{cccccccc}
0 & 0 & 0 & 0 & 0 & 0 & 0 & -1 \\
0 & 0 & 0 & 0 & 0 & -1 & 0 & 0 \\
0 & 0 & 0 & 0 & 0 & -1 & -1 & 0 \\
0 & 0 & 0 & 0 & -1 & 0 & 0 & 0 \\
0 & 0 & 0 & 1 & 0 & 0 & 0 & 0 \\
0 & 1 & 1 & 0 & 0 & 0 & h_{1} & 3 k_{1}-h_{2} \\
0 & 0 & 1 & 0 & 0 & -h_{1} & 0 & -2 h_{2} \\
1 & 0 & 0 & 0 & 0 & -3 k_{1}+h_{2} & 2 h_{2} & 0
\end{array}\right]
$$




$$
\widehat{P}_{1}=\left[\begin{array}{cccccccc}
0 & 0 & 1 & 0 & 0 & 0 & h_{1} & k_{1}-2 h_{2} \\
& 0 & 0 & 0 & 0 & -k_{1} & 0 & -k_{2} \\
& & 0 & 0 & -2 h_{1} & -k_{1} & -h_{2} & -k_{2} \\
& & & 0 & -k_{1} & -k_{2} & -k_{2} & 0 \\
& & & 0 & 0 & h_{3}+h_{1}^{2} & -2 h_{4}+k_{3}+2 h_{1} k_{1}-2 h_{2} h_{1} \\
& & & & 0 & h_{1} k_{1} & 3 k_{1}^{2}-h_{2} k_{1} \\
& 0 & & & 0 & g_{1}-h_{1} k_{2}-k_{1}^{2}-h_{2}^{2} \\
& & & & & & 0
\end{array}\right] .
$$

Тензор Нийенхейса в присоединенном представлении имеет вид

$$
N^{*}=\left[\begin{array}{cccccccc}
-k_{1} & k_{2} & k_{2} & 0 & N^{1,5} & 0 & N^{1,7} & 3 k_{1} k_{2}-k_{2} h_{2} \\
0 & k_{1} & 4 k_{1} & 0 & -h_{1}^{2}+h_{3} & 0 & N^{2,7} & N^{2,8} \\
-h_{1} & 0 & -h_{2} & k_{2} & -h_{3}-h_{1}^{2} & 0 & N^{3,7} & N^{3,8} \\
0 & 0 & 2 h_{1} & k_{1} & 0 & 0 & h_{3}+h_{1}{ }^{2} & N^{4,8} \\
0 & 0 & 0 & 0 & k_{1} & k_{2} & k_{2} & 0 \\
0 & 0 & 0 & 0 & 0 & k_{1} & 0 & k_{2} \\
1 & 0 & 0 & 0 & 2 h_{1} & 0 & h_{2} & 0 \\
0 & 0 & -1 & 0 & 0 & 0 & -h_{1} & 2 h_{2}-k_{1}
\end{array}\right],
$$

где

$$
\begin{aligned}
& N^{1,5}=-2 h_{2} h_{1}+2 h_{4}-k_{3}-2 h_{1} k_{1}, \quad N^{1,7}=-h_{2}^{2}-g_{1}+h_{1} k_{2}+k_{1}^{2}, \\
& N^{2,7}=4 h_{1} k_{1}, \quad N^{2,8}=-6 h_{2} k_{1}-h_{2}^{2}+7 k_{1}^{2}-g_{1}, \\
& N^{3,7}=-2 h_{2} h_{1}, \quad N^{3,8}=3 h_{2}^{2}-2 h_{2} k_{1}+g_{1}-k_{1}{ }^{2}, \\
& N^{4,8}=-2 h_{4}+k_{3}+2 h_{1} k_{1}-2 h_{2} h_{1} .
\end{aligned}
$$

Разделение переменных является результатом спектрального анализа матрицы $N^{*}$ и связано с введением некоторых специальных множеств координат, которые мы рассмотрим в следующем пункте.

4.2. Координаты Дарбу-Нийенхейса и Ганкеля-Фробениуса. Пусть $\left(M, P_{0}, N=P_{1} P_{0}^{-1}\right)-$ ПН-многообразие размерности $2 n$. В работе [1] было доказано, что если $N$ имеет $n$ функционально независимых собственных значений $\left(\lambda_{1}, \ldots, \lambda_{n}\right)$ (иначе говоря, $N$ максимально), можно ввести множество координат $\left(\boldsymbol{\lambda}:=\left(\lambda_{1}, \ldots, \lambda_{n}\right)\right.$; $\left.\boldsymbol{\mu}:=\left(\mu_{1}, \ldots, \mu_{n}\right)\right)$, таких что $P_{0}$ и $N$ принимают вид

$$
P_{0}=\left[\begin{array}{cc}
0 & \boldsymbol{I} \\
-\boldsymbol{I} & 0
\end{array}\right], \quad N=\left[\begin{array}{cc}
\boldsymbol{\Lambda} & 0 \\
0 & \boldsymbol{\Lambda}
\end{array}\right],
$$

где $\boldsymbol{I}$ - единичная $(n \times n)$-матрица, а $\boldsymbol{\Lambda}:=\operatorname{diag}\left(\lambda_{1}, \ldots, \lambda_{n}\right)$. В работе [1] было также отмечено, что когда первые $n$ координат являются собственными значениями $N$, оставшиеся координаты можно построить в квадратурах. Поскольку эти координаты являются каноническими по отношению к $P_{0}$ и диагонализуют тензор Нийенхейса, о них можно говорить как о координатах Дарбу-Нийенхейса (ДН). 
Полезно ввести другое множество координат, которое мы обозначим как $(\boldsymbol{f}:=$ $\left.\left(f_{1}, \ldots, f_{n}\right) ; \boldsymbol{c}:=\left(c_{1}, \ldots, c_{n}\right)\right)$. Идея заключается в следуюшем: коэффишиенты $c_{j}$ минимального многочлена тензора Нийенхейса

$$
(\operatorname{det}(\lambda-N))^{\frac{1}{2}}=\lambda^{n}-\sum_{i=1}^{n} c_{i} \lambda^{n-i}
$$

удовлетворяют следуюшему замечательному свойству:

$$
\begin{aligned}
& N^{*} d c_{1}=d c_{2}+c_{1} d c_{1}, \\
& N^{*} d c_{2}=d c_{3}+c_{2} d c_{1}, \\
& \ldots \ldots \ldots \ldots \ldots \ldots \\
& N^{*} d c_{n}=c_{n} d c_{1} .
\end{aligned}
$$

Аналогичным образом будем искать множество дополнительных переменных $\left\{f_{1}, \ldots\right.$ $\left.\ldots, f_{n}\right\}$, таких что:

1) их дифференциалы порождают $n$-мерное распределение в $T^{*} S_{0}$, дополнительное к распределению, порождаемому дифференциалами $d c_{i}$;

2) они удовлетворяют тем же рекуррентным соотношениям

$$
\begin{aligned}
& N^{*} d f_{1}=d f_{2}+c_{1} d f_{1}, \\
& N^{*} d f_{2}=d f_{3}+c_{2} d f_{1}, \\
& \ldots \ldots \ldots \ldots \ldots \ldots \\
& N^{*} d f_{n}=c_{n} d f_{1} .
\end{aligned}
$$

Характерной особенностью координат $(\boldsymbol{f} ; \boldsymbol{c})$ является то, что $P_{0}$ и $N^{*}$ принимают матричный вид

где

$$
P_{0}=\left[\begin{array}{cc}
0 & \boldsymbol{H} \\
-\boldsymbol{H}^{T} & 0
\end{array}\right], \quad N^{*}=\left[\begin{array}{cc}
\boldsymbol{F} & 0 \\
0 & \boldsymbol{F}
\end{array}\right],
$$

$$
\boldsymbol{H}=\left[\begin{array}{cccc}
0 & 0 & \ldots & 1 \\
0 & \ldots & 1 & -c_{1} \\
1 & -c_{1} & \ldots & -c_{n-1}
\end{array}\right], \quad \boldsymbol{F}=\left[\begin{array}{cccc}
c_{1} & c_{2} & \ldots & c_{n} \\
1 & 0 & \ldots & 0 \\
0 & \ldots & 1 & 0
\end{array}\right],
$$

откуда следует определение координат Ганкеля-Фробениуса (ГФ).

Нетрудно показать, что соотношение между ГФ-координатами $(\boldsymbol{f} ; \boldsymbol{c})$ и ДН-координатами $(\boldsymbol{\lambda} ; \boldsymbol{\mu})$ дается системой

$$
\begin{aligned}
& \lambda^{n}-\sum_{i=1}^{n} c_{i} \lambda^{n-i}=\prod_{i=1}^{n}\left(\lambda-\lambda_{i}\right), \\
& \mu_{k}=\sum_{i=1}^{n} f_{i} \lambda_{k}^{n-i} \quad(k=1, \ldots, n) .
\end{aligned}
$$

Преимушество использования системы $Г \Phi$-координат состоит в том, что в нашем случае их можно вычислить алгебраическим способом из рекуррентных соотношений (4.9).

В заключение заметим, что благодаря последним уравнениям из (4.8) и (4.9), гамильтоновы поля $X=P_{0} d c_{1}$ и $Y=P_{0} d f_{1}$ являются пфаффиановыми квазибигамильтоновыми векторными полями в терминологии работ [20, 2]. Таким образом, эти аргументы доказывают сушествование таких полей на любом максимальном ПН-многообразии.

3 Теоретическая и математическая физика, т. 122, № 2, 2000 г. 
4.3. Назад к $\mathbf{B s q}_{5}$. Вычисляя минимальный многочлен матрицы $N^{*}(4.5)$, получаем координаты $\boldsymbol{c}$ :

$$
\begin{aligned}
& c_{1}=k_{1}+h_{2}, \quad c_{2}=2 k_{1}^{2}-h_{2}^{2}-g_{1}+2 h_{1} k_{2}-h_{2} k_{1}, \\
& c_{3}=-3 k_{1}^{3}+2 k_{1} h_{2}^{2}+2 k_{1} g_{1}-2 h_{1} k_{1} k_{2}-2 h_{1} h_{2} k_{2}+2 k_{2} h_{4}-k_{3} k_{2}-h_{2} k_{1}^{2}, \\
& c_{4}=k_{2}^{2} h_{3}-g_{1} k_{1}^{2}+h_{2} k_{1}^{3}-h_{1}^{2} k_{2}^{2}+k_{1}^{4}+2 h_{2} h_{1} k_{1} k_{2}-2 k_{1} h_{2} h_{4}+k_{1} k_{2} k_{3}-h_{2}^{2} k_{1}^{2} .
\end{aligned}
$$

Координаты $f$ можно найти следующим образом. Учитывая свойства однородности теории с весами $[\lambda]=3,\left[h_{i}\right]=1+i,\left[k_{i}\right]=2+i$, мы видим, что $\left[P_{0}\right]=-8$ и $\left[c_{i}\right]=3 i$. Из уравнения (4.11) получаем, что вес $f_{1}$ должен быть равен -4 . Взяв анзац $f_{1}=1 / k_{2}$ и используя рекуррентные соотношения (4.9), находим координаты $f$ :

$$
\begin{gathered}
f_{1}=\frac{1}{k_{2}}, \quad f_{2}=\frac{-h_{2}}{k_{2}}, \quad f_{3}=-h_{1}+\frac{g_{1}+h_{2}^{2}-2 k_{1}^{2}}{k_{2}}, \\
f_{4}=k_{3}-h_{4}+h_{1} h_{2}+\frac{k_{1}^{3}+h_{2} k_{1}^{2}-k_{1} h_{2}^{2}-g_{1} k_{1}}{k_{2}} .
\end{gathered}
$$

ГФ-координаты, ассоциированные с ПН-структурой, позволяют реконструировать спектральную кривую (3.12) и уравнения Склянина для разделения переменных [21]. Аналогично тому, как это сделано в случае уравнения $\mathrm{K} д \Phi[3,6]$, рассмотрим уравнения (4.12) как определяюшие уравнения для двух фундаментальных полиномов, ассоциированных с задачей,

$$
\mathcal{Q}_{1}=\lambda^{4}-\sum_{i=1}^{4} c_{i} \lambda^{(4-i)}, \quad \mathcal{Q}_{2}=\sum_{i=1}^{4} f_{i} \lambda^{(4-i)} .
$$

Теперь прямыми выгислениями можно показать, что в нулях $\lambda_{j}$ полинома $\mathcal{Q}_{1}$ и при соответствуюших значениях $\mu_{j}=\mathcal{Q}_{2}\left(\lambda_{j}\right)$ выполняется уравнение спектральной кривой (3.12):

$$
\mu_{j}^{3}=\mu_{j}\left(H_{1} \lambda_{j}+H_{2}\right)+\left(\lambda_{j}^{5}+H_{3} \lambda_{j}^{3}+H_{4} \lambda_{j}^{2}+H_{5} \lambda_{j}+H_{6}\right) .
$$

При ограничении задачи на лист $S_{0}$ рассмотрим $H_{1}=v_{1}$ и $H_{3}=v_{2}$ как фиксированные параметры. Отсюда получим уравнения Склянина для разделения переменных [21], т.е. четыре соотношения в канонических координатах $(\boldsymbol{\lambda} ; \boldsymbol{\mu})$, зависяшие от четырех коммутируюших гамильтонианов $\widehat{H}_{2}, \widehat{H}_{4}, \widehat{H}_{5}, \widehat{H}_{6}$. Как хорошо известно, по аналогии с классической теорией Штэкеля разделения переменных для гамильтонианов, квадратично зависящих от импульсов, из этих соотношений следует, что полный интеграл $W=W_{1}+W_{2}+W_{3}+W_{4}$ уравнений Гамильтона-Якоби

$$
\widehat{H}_{i}\left(\boldsymbol{\lambda}, \frac{\partial W}{\partial \boldsymbol{\lambda}}\right)=\alpha_{i}, \quad i=2,4,5,6,
$$

получается путем решения четырех расцепленных обыкновенных дифференциальных уравнений первого порядка

$$
\left(\frac{d W_{j}}{d \lambda_{j}}\right)^{3}=\frac{d W_{j}}{d \lambda_{j}}\left(v_{1} \lambda_{j}+\alpha_{2}\right)+\left(\lambda_{j}^{5}+v_{2} \lambda_{j}^{3}+\alpha_{4} \lambda_{j}^{2}+\alpha_{5} \lambda_{j}+\alpha_{6}\right), \quad j=1, \ldots, 4,
$$

что представляет собой задачу, которую можно исследовать алгебро-геометрическими методами. 


\section{5. ЦЕПОЧКИ НИЙЕНХЕЙСА И РАЗДЕЛЕНИЕ ПЕРЕМЕННЫХ ЛЕВИ-ЧИВИТА}

В этом разделе мы вводим концепцию цепочек Нийенхейса как естественного расширения последовательностей Ленарда и приводим альтернативное и несколько более классическое доказательство того факта, что ДН-координаты являются разделяющимися переменными для уравнения Гамильтона-Якоби, соответствующего каждому гамильтонову векторному полю $\widehat{X}_{j}$ нашей задачи. Отметим, что когда мы имели дело с алгебро-геометрическим разделением переменных в разделе 4 , исходные гамильтонианы (3.13) 10-мерной задачи Гельфанда-Захаревича (см. предложение 2) не использовались при построении ДН- и ГФ-координат, а входили в доказательство разделимости переменных только на завершающей стадии. Причина этого в том, что будучи неинвариантными относительно “распределения" $\widehat{\mathcal{D}}$, они не определяют рекуррентное соотношение Ленарда на $S_{0}$, по крайней мере при классическом рассмотрении, как, например, в работах $[12,22]$. Мы рассмотрим их ограничение $\widehat{H}_{j}$ на симплектический лист $S_{0}$ в соответствии со следующим критерием разделимости, предложенным Бененти [23]:

ПРЕДЛОЖЕНИЕ 4. Пусть $X=P_{0} d K$ - гамильтоново векторное поле на симплектическом многообразии $\left(M, \omega=P_{0}^{-1}\right), \quad$ a $(\boldsymbol{q} ; \boldsymbol{p}) \quad\left(\boldsymbol{q}:=\left(q_{1}, \ldots, q_{n}\right) ; \quad \boldsymbol{p}:=\right.$ $\left.\left(p_{1}, \ldots, p_{n}\right)\right)$ - набор координат Дарбу. Эти координаты являются разделяющимися переменными для соответствующего уравнения Гамильтона-Якоби тогда и только тогда, когда $X$ допускает $n$ интегралов движения $\left\{K_{i}\right\}_{1 \leqslant i \leqslant n}$, таких чmo

$$
\operatorname{det}\left(\frac{\partial K_{i}}{\partial p_{j}}\right) \neq 0
$$

и которые находятся в разделяющейся инволюиии по отношению $\kappa(\boldsymbol{q} ; \boldsymbol{p})$, т.е. удовлетворяют п соотношениям

$$
\left.\left\{K_{i}, K_{j}\right\}\right|_{k}:=\frac{\partial K_{i}}{\partial q_{k}} \frac{\partial K_{j}}{\partial p_{k}}-\frac{\partial K_{i}}{\partial p_{k}} \frac{\partial K_{j}}{\partial q_{k}}=0
$$

где не производится суммирование по $k$.

Для того чтобы использовать этот критерий, введем понятие цепочки Нийенхейса, обобшаюшее классическое рекуррентное соотношение Ленарда, которому удовлетворяют (нормированные) следы степеней тензора Нийенхейса $I_{k}=(1 / 2 k) \operatorname{Tr} N^{k}$,

$$
N^{*} d I_{k}=d I_{k+1} \Rightarrow\left(N^{*}\right)^{k-1} d I_{1}=d I_{k}
$$

и соотношения, которым удовлетворяют ГФ-координаты $(\boldsymbol{f} ; \boldsymbol{c})$ вследствие уравнений (4.8), (4.9).

ОПРЕДЕЛЕНИЕ. Пусть $(M, P, N)$ - ПН-многообразие, а $\left\{K_{j}\right\}_{1 \leqslant j \leqslant n}-n$ независимых функций, обладаюших следуюшим свойством по отношению к $N^{*}$ :

$$
d K_{j}=\sum_{k=1}^{n} a_{j k}\left(N^{*}\right)^{k-1} d K_{1}, \quad j=1, \ldots, n
$$


где $a_{j k}$ - элементы обратимой матрично-значной функции $\boldsymbol{a}$. Об этом рекуррентном соотношении (и о соответствуюшем соотношении $X_{j}=P d K_{j}$ для векторных полей) мы будем говорить как о цепочке Нийенхейса с генератором $K_{1}\left(X_{1}=P d K_{1}\right)$.

Цепочки Нийенхейса были неявным образом рассмотрены в работе [24], где было доказано, что функции $K_{j}$, допускающие представление (5.3), находятся в инволюции по отношению к пуассонову тензору $P$. В настояшей работе мы хотим констатировать более сильное свойство таких функций, а именно то, что они находятся в разделяющейcs инволюции по отношению к ДН-координатам. Это легко доказать, если вычислить $\left.\left\{K_{i}, K_{j}\right\}\right|_{k}$ в ДН-координатах и вспомнить, что $\lambda_{j}$ являются собственными значениями тензора $N^{*}$. Таким образом, в силу предложения 4 мы можем сделать следующее утверждение:

ПРЕДЛОЖЕНИЕ 5. ДН-координаты являются разделяющимися переменными для каждой функции $K_{j}$ из чепочки Нийенхейса.

Таким образом, для доказательства того, что Bsq5-система удовлетворяет теореме Бененти, достаточно проверить, что ограничения $\widehat{H}_{j}$ гамильтонианов $H_{j}$ на симплектический лист $S_{0}$ обшего положения принадлежат цепочке Нийенхейса. Можно непосредственно проверить, что это имеет место: действительно, выбирая в качестве генератора $K_{1}$ гамильтониан

$$
\begin{aligned}
\widehat{H}_{2}= & k_{3}{ }^{2}-h_{4} k_{3}+h_{3} k_{1}{ }^{2}+h_{2}{ }^{2} h_{3}-2 h_{1} k_{2}{ }^{2}-3 k_{1}{ }^{2} k_{2}+h_{4}{ }^{2}- \\
& -h_{1} k_{1} k_{3}+h_{2} h_{1} k_{3}+h_{1} k_{2} h_{3}-2 h_{1} h_{2} h_{4}-k_{1} k_{2} h_{2}-2 k_{1} h_{2} h_{3}+ \\
& +2 k_{1} h_{1} h_{4}-k_{1} v_{2}+k_{2} g_{1}-k_{2} v_{1}+h_{3} g_{1}-h_{1}{ }^{2} g_{1},
\end{aligned}
$$

где отождествлено $K_{2}=\widehat{H}_{4}, K_{3}=\widehat{H}_{5}, K_{4}=\widehat{H}_{6}$, получаем для матрицы $\boldsymbol{a}$ в рекуррентном соотношении Нийенхейса (в координатах $(\boldsymbol{f} ; \boldsymbol{c})$ ) выражение

$$
\boldsymbol{a}=\left[\begin{array}{cccc}
1 & 0 & 0 & 0 \\
-f_{1} c_{1}-f_{2} & f_{1} & 0 & 0 \\
-f_{1} c_{2}-f_{3} & -f_{1} c_{1} & f_{1} & 0 \\
-f_{1} c_{3}-f_{4} & -c_{2} f_{1} & -f_{1} c_{1} & f_{1}
\end{array}\right]
$$

\section{6. ЗАКЛЮЧЕНИЕ}

На конкретном примере стационарной Bsq5-системы мы обсудили два связанных между собой вопроса. Сначала мы обрисовали систематический путь получения стационарных редукций и их гамильтоновых структур для $n$-теорий Гельфанда-Дикого. Ключевые моменты состоят в изучении ЦС (2.4) и ее стационарных многообразий, а также представления нулевой кривизны и представления Лакса (3.2), естественным образом получающихся из потоков. Гамильтоновы структуры индуцируются на стационарном многообразии при помоши специальной процедуры бигамильтоновой редукции для па- 
ры пуассоновых структур, определенных на пространстве полиномов от спектрального параметра $\lambda$ со значениями в алгебре Ли. Такая процедура, обоснование которой базируется на связи между формулировками Лакса и Пуассона для гамильтоновых потоков, позволяет нам отнести такие теории к классу теорий Гельфанда-Захаревича вырожденных пучков пуассоновых структур, вообще говоря (т.е. при $n>2$ ), не максимального ранга (см. раздел 3 ).

В разделах 4 и 5 мы исследовали способы решения этих интегрируемых динамических систем с помошью метода разделения переменных. Нашим основным сюжетом было изучение некоторых соотношений между классической теорией Гамильтона-Якоби (а также ее более современных аспектов, связанных с понятием полной алгебраической интегрируемости) и гамильтоновой структурой, полученной в разделе 3 . С этой целью мы отказались от теории Гельфанда-Захаревича и при помощи дальнейшей (но уже не бигамильтоновой) редукции установили связь с теорией ПН-многообразий. Мы исследовали свойства двух примечательных классов систем координат, ассоциированных с ПН-структурами - ДН- и ГФ-координаты. Используя эти координаты, мы доказали разделяемость Bsq5-потоков двумя способами. В первом способе мы явно установили связь с “методом полюсов функции Бейкера-Ахиезера", разработанным российской школой (см., например, [6, 21]). Во втором способе мы ввели понятие цепочек Нийенхейса и доказали разделяемость, используя условие, эквивалентное классическому критерию разделяемости Леви-Чивита [25].

В заключение заметим, что особенность второй редукции, которая все еше попадает в класс МР-редукций, заключается в том, что два различных геометрических процесса используются одновременно: ограничение для векторных полей и проекция для бигамильтоновых структур. Из-за этого мы сохранили бигамильтонову структуру (в виде ПН-структуры), но потеряли бигамильтонову формулировку для векторных полей. Эта ситуация не является новой в гамильтоновой теории интегрируемых систем - такое происходит, например, в случае интегрируемой системы Хенона-Хейлеса и ее многомерных обобщений, получающихся путем редукции из стационарных потоков КдФ-иерархии $[24,26]$, и для широкого класса потенциалов, восстановленных из ограниченных потоков связанных КдФ-систем, наиболее представительные члены которых - это системы Гарнье [27].

Такие классы динамических систем определены на бигамильтоновом многообразии $M$ максимального ранга, и их формулировку Нийенхейса, а именно специальный случай, называемый квазибигамильтоновой формулировкой $[20,28]$, можно получить после аналогичной редукции. Стационарные потоки иерархии Буссинеска (и теорий Гельфанда-Дикого с более высокими $n$ ) представляют собой примеры бигамильтоновых структур немаксимального ранга, для которых анализ, аналогичный проведенному в работе [7] для пуассоновых пучков максимального ранга, насколько нам известно, в настояшее время отсутствует.

Благодарности. Мы благодарим М. Педрони и П. Сазати за полезные дискуссии. Г.Ф. и Г.Т. хотели бы поблагодарить организаторов конференции NEEDS в Лидсе в 1998 г. за предложение представить полученные результаты. Большинство вычислений было проделано с помощью программы Maple $\mathrm{V}^{\circledR}$. Работа выполнена при поддержке G.N.F.M. итальянского C.N.R. 


\section{Список литературы}

[1] F. Magri, T. Marsico. Some developments of the concepts of Poisson manifolds in the sense of A. Lichnerowicz. In: Gravitation, Electromagnetism, and Geometric Structures. Ed. G. Ferrarese. Bologna: Pitagora Editrice, 1996. P. 207-222; T. Marsico. Una caratterizzazione geometrica dei sistemi che ammettono rappresentazione alla Lax estesa. Tesi di Dottorato. Università di Milano, 1996.

[2] C. Morosi, G. Tondo. J. Phys. A. 1997. V. 30. P. 2799-2806.

[3] S. I. Alber. Commun. Pure Appl. Math. 1981. V. 34. P. 259-272.

[4] M. Antonowicz, A. P. Fordy, S. Wojciechowski. Phys. Lett. A. 1987. V. 124. P. 455-462.

[5] О. И. Богоявленский, С. П. Новиков. Функц. анализ и его прилож. 1976. Т. 10. № 1. C. $9-13$.

[6] Б. А. Дубровин, И. М. Кричевер, С. П. Новиков. Интегрируемые системы. І. В сб.: Итоги науки и техники. Современные проблемы математики. Фундаментальные направления. Т. 4. Динамические системы - 4. Ред. Р. В. Гамкрелидзе. М.: ВИНИТИ, 1985. С. 179-285.

[7] I. M. Gel'fand, I. Zakharevich. On the local geometry of a bi-Hamiltonian structure. In: The Gelfand Mathematical Seminars 1990-1992. Eds. L. Corwin et al. Boston: Birkhäuser, 1993. P. 51-112.

[8] P. Casati, G. Falqui, F. Magri, M. Pedroni. J. Math. Phys. 1997. V. 38. P. 4605-4628.

[9] G. Falqui, F. Magri, M. Pedroni. Commun. Math. Phys. 1998. V. 197. P. 303-324.

[10] M. Sato, Y. Sato. Soliton equations as dynamical systems on infinite-dimensional Grassmann manifold. In: Nonlinear PDEs in Applied Sciences (US-Japan Seminar, Tokyo). Eds. P. Lax, H. Fujita. Amsterdam: North-Holland Publ. Company, 1982. P. 259-271.

[11] L. A. Dickey. Soliton Equations and Hamiltonian Systems. Adv. Ser. in Math. Phys. V. 12. Singapore: World Scientific, 1991.

[12] F. Magri, C. Morosi. A geometric characterization of integrable Hamiltonian systems through the theory of Poisson-Nijenhuis manifolds. Quaderno S/19, Università di Milano, 1984.

[13] А. Г. Рейман, М. А. Семенов-Тянь-Шанский. Теоретико-групповые методы в теории интегрируемых систем. В сб.: Итоги науки и техники. Современные проблемы математики. Фундаментальные направления. Т. 16. Динамические системы - 7. Ред. Р. В. Гамкрелидзе. М.: ВИНИТИ, 1987. С. 119-194.

[14] G. Magnano, F. Magri. Rev. Math. Phys. 1991. V. 3. P. 403-466.

[15] M. Pedroni, P. Vanhaecke. Reg. Chaotic Dyn. 1998. V. 3. P. 132-160.

[16] J. E. Marsden, T. Ratiu. Lett. Math. Phys. 1986. V. 11. P. 161-169.

[17] P. Casati, F. Magri, M. Pedroni. Bihamiltonian manifolds and $\tau$-function. In: Mathematical Aspects of Classical Field Theory. Contemp. Math. V. 132. Eds. M. J. Gotay et al. Providence, R.I.: American Mathematical Society, 1991. P. 213-234.

[18] A. P. Fordy, S. D. Harris. Hamiltonian structures in stationary manifold coordinates. In: Algebraic Aspects of Integrable Systems. Progr. Nonlinear Differ. Equ. Appl. V. 26. Eds. A. S. Fokas, I. M. Gelfand. Boston, MA: Birkhauser, 1996. P. 103-130.

[19] A. Fokas, B. Fuchssteiner. Physica D. 1981. V. 4. P. 47-66.

[20] R. Brouzet, R. Caboz, J. Rabenivo, V. Ravoson. J. Phys. A. 1996. V. 29. P. 2069-2076.

[21] E. Sklyanin. Progr. Theor. Phys. Suppl. 1995. V. 118. P. 35-60.

[22] S. De Filippo, G. Marmo, M. Salerno, G. Vilasi. Nuovo Cimento B. 1984. V. 83. P. 97-112.

[23] S. Benenti. Separability structures on Riemannian manifolds. In: Lect. Notes Math. V. 836. Differential Geometrical Methods in Mathematical Physics. Eds. P. L. García, A. Péres-Rendón, and J. M. Sourian. Berlin: Springer, 1980. P. 512-538.

[24] G. Tondo. J. Phys. A. 1995. V. 28. P. 5097-5115.

[25] T. Levi-Civita. Math. Annal. 1904. V. 59. P. 383-397.

[26] G. Tondo. On the integrability of Hénon-Heiles type systems. In: Non Linear Physics, Theory and Experiment. Eds. E. Alfinito et al. Singapore: World Scientific, 1996. P. 313-320.

[27] M. Btaszak. Multi-Hamiltonian Theory of Dynamical Systems. Berlin: Springer, 1998.

[28] C. Morosi, G. Tondo. To be published in Rep. Math. Phys. 